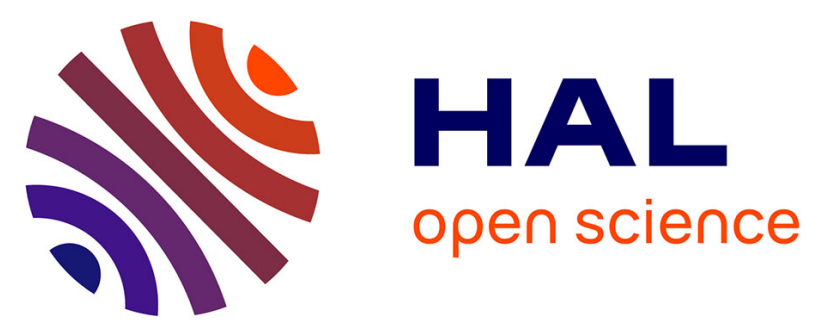

\title{
Réintégrer sans modifier les hiérarchies coloniales ? Inégalités ethniques et territoriales dans les politiques d'assistance aux mutilés de guerre de l'Empire colonial français (1916-1939)
}

Gildas Brégain

\section{To cite this version:}

Gildas Brégain. Réintégrer sans modifier les hiérarchies coloniales ? Inégalités ethniques et territoriales dans les politiques d'assistance aux mutilés de guerre de l'Empire colonial français (1916-1939). Alter: European Journal of Disability Research / Revue européenne de recherche sur le handicap, 2019, 13 (4), pp.263-281. 10.1016/j.alter.2019.09.002 . halshs-02314435

\author{
HAL Id: halshs-02314435 \\ https://shs.hal.science/halshs-02314435
}

Submitted on 12 Oct 2019

HAL is a multi-disciplinary open access archive for the deposit and dissemination of scientific research documents, whether they are published or not. The documents may come from teaching and research institutions in France or abroad, or from public or private research centers.
L'archive ouverte pluridisciplinaire HAL, est destinée au dépôt et à la diffusion de documents scientifiques de niveau recherche, publiés ou non, émanant des établissements d'enseignement et de recherche français ou étrangers, des laboratoires publics ou privés. 


\section{Réintégrer sans modifier les hiérarchies coloniales ? Inégalités ethniques et territoriales dans les politiques d'assistance aux mutilés de guerre de l'Empire colonial français (1916-1939).}

Gildas Brégain, chercheur CNRS, Univ. Rennes, Arenes - UMR 6051, F. 35000 Rennes, France.

\section{Résumé}

Cet article s'intéresse aux politiques d'assistance aux mutilés de guerre de l'Empire colonial français. Ces politiques sont le résultat d'un ensemble d'actions et d'interactions entre de multiples acteurs, à savoir les ministères (Colonies, Affaires Etrangères, Travail, Pensions), les parlementaires, l'Office National des Mutilés, les associations de mutilés de guerre et les hauts fonctionnaires coloniaux. Fondé sur de multiples archives, sur les revues associatives et la presse coloniale, cet article analyse le statut concédé aux mutilés de guerre de ces territoires. Constituent-ils, du fait de leur sacrifice pour la Patrie, des "créanciers" égaux à ceux de la métropole ? Nos recherches permettent de dresser le constat d'une extrême hétérogénéité des politiques d'assistance dans l'Empire colonial, avec de fortes inégalités territoriales et ethniques dans l'attribution des différentes prestations. Les mutilés de guerre de l'Empire bénéficient d'une partie non négligeable des droits conférés à ceux de la métropole (y compris les droits économiques). Les mutilés français et indigènes d'Afrique du Nord et des quatre communes du Sénégal bénéficient d'une pension relativement similaire à celle des mutilés en France. Dans les autres colonies, les mutilés indigènes sont discriminés et perçoivent des pensions d'un montant très inférieur. Dans tous les territoires de l'Empire, les mutilés indigènes ont un accès restreint aux emplois administratifs et aux prêts agricoles. Cette politique sociale, coûteuse pour la métropole, s'avère prioritaire en raison de l'impératif politique de reconnaissance envers ceux qui se sont sacrifiés pour la patrie, mais aussi et surtout pour conserver la sympathie des populations colonisées et le soutien politique des mutilés et des anciens combattants dans un contexte de nationalisme anticolonial croissant.

Mots clefs : politique sociale impériale, mutilés de guerre, colonialisme, droits, handicap

\section{Introduction}

Comme l'a montré Antoine Prost pour la métropole française, les associations d'anciens combattants constituent un acteur politique et social incontournable pendant l'entredeux-guerres, alliant un réseau dense d'associations et une position morale à laquelle il est difficile de résister publiquement (Prost, 1977). En effet, les responsables politiques admettent que les anciens combattants détiennent des droits sacrés du fait de leurs sacrifices au nom de la Patrie. Possédant des marques physiques indélébiles de leurs sacrifices, les mutilés de guerre de la métropole obtiennent des avantages économiques et sociaux considérables. Outre le droit à une pension pour invalidité (établie par la loi du 13 mars 1919), ils bénéficient d'un accès privilégié à un emploi : d'abord à des «emplois réservés » dans les administrations publiques (loi du 17 avril 1916), à un accès préférentiel à des emplois réservés de l'État, des établissements publics et des communes (loi du 30 janvier 1923) (Bette, 2006), puis ils bénéficient ensuite de l'obligation d'emploi d'un quota de $10 \%$ de mutilés dans les entreprises privées (loi du 26 avril 1924) (Romien, 2005). Cette loi autorise les employeurs à diminuer la 
rémunération des mutilés en cas de diminution de leur rendement, tout en assurant à l'invalide la garantie d'un salaire au moins égal à la moitié du salaire normal. D'autres dispositions législatives sont adoptées pour organiser la rééducation professionnelle et créer un Office national des mutilés et réformés de la guerre (loi du 2 janvier 1918). Les autorités et les parlementaires envisagent une application différée et différenciée de ces législations dans les territoires de l'Empire colonial.

En France, plusieurs études ont porté sur les mutilés de la Première Guerre mondiale, centrées soit sur les politiques de réinsertion professionnelle (Romien, 2005 ; Fichou, 2014) ; soit sur le fonctionnement d'une institution de rééducation professionnelle en province et ses bénéficiaires, en majorité cultivateurs, ouvriers et petits artisans (Collard, 2018) soit sur certaines catégories de mutilés comme les blessés de la face (Delaporte, 2001). Cependant, ces études se sont focalisées sur la métropole et ont ignoré le sort des mutilés originaires de l'Empire colonial, largement minoritaires par rapport aux mutilés de la métropole, qui sont près d'un million. En l'absence de statistiques précises, nous estimons le nombre de mutilés dans l'Empire entre 25000 et 50 000, présents en majorité dans les territoires d'Afrique du Nord (entre 10000 et 20 000), en Afrique-Occidentale française ${ }^{1}$ (AOF) (environ 11000). Cette répartition géographique est à mettre en regard avec les effectifs envoyés par chaque territoire colonial pour combattre sur les fronts. Les pays d'Afrique du Nord (Algérie en premier lieu, Tunisie et Maroc), et les territoires de l'AOF envoient respectivement près de 275000 et 170000 combattants sur les fronts. Madagascar et les Antilles en envoient plusieurs dizaines de milliers.

Cet article vise à combler ce vide historiographique en s'interrogeant sur les politiques d'assistance aux mutilés de guerre dans les divers territoires de l'Empire. Il contribue ainsi à renforcer une dynamique de recherche déjà engagée en faveur des «combattants de l'Empire » (Fremeaux, 2006). Si l'on reprend la définition de Jane Burbank et Frederick Cooper, l'Empire colonial français constitue une « vast[e] unit[é] politiqu[e], expansionnist[e] ou conservant le souvenir d'un pouvoir étendu dans l'espace, qui maintien[t] la distinction et la hiérarchie à mesure qu'ell[e] incorpor[e] de nouvelles populations » (Burbank et Cooper, 2011). Les territoires de l'Empire français relèvent de statuts administratifs distincts (anciennes et nouvelles colonies, protectorat, mandat), et les populations résidentes sont traitées de manière différenciée (français/étrangers/indigènes), en se fondant sur des conceptions racistes légitimées sur le plan juridique (code de l'indigénat).

Les politiques d'assistance aux mutilés sont le résultat d'un « ensemble d'actions, de décisions, d'interactions et de rapports de force évolutifs » (Lagroye, François, Sawicki, 2006, p. 507) entre de multiples acteurs qui possèdent des ressources extrêmement inégales : les ministères (Guerre, Colonies, Affaires étrangères, Travail, Pensions) ; les parlementaires ; l'Office National des Mutilés (puis Office National des Mutilés et Réformés, ONMR); les associations de mutilés de guerre et les hauts fonctionnaires coloniaux. En prêtant attention à la diversité des populations résidentes et des territoires de l'Empire, cet article prétend analyser le statut concédé aux mutilés de guerre de ces territoires. Constituent-ils, du fait de

\footnotetext{
${ }^{1}$ Pendant les années 1920, l'Afrique-Occidentale française regroupe huit colonies françaises au sein d'une même fédération : la Mauritanie, le Sénégal, le Soudan français, la Guinée française, la Côte d'Ivoire, le Niger, la Haute-Volta et le Dahomey. L’AOF est dirigée par un gouverneur général présent à Dakar.
} 
leur sacrifice pour la Patrie, des créanciers égaux à ceux de la métropole ? L'impératif de traiter dignement les mutilés indigènes conduit-il les autorités à revoir les hiérarchies coloniales et la distinction entre citoyens français et sujets indigènes ?

Pour mieux cerner les enjeux de ces politiques d'assistance, il convient de prendre en compte les circulations intra-impériales des hommes (soldats, dirigeants associatifs, responsables politiques), des idées (notion de droits des mutilés), de l'argent (pensions), et des objets (prothèses). Ces circulations témoignent de l'intérêt porté par les autorités aux mutilés de l'Empire, et conditionnent la nature des prestations qui leur sont délivrées. Notre étude débute en 1916, lorsque ces soldats mutilés commencent à revenir massivement dans leurs pays d'origine, et se termine en 1939, lorsque débute la Seconde Guerre mondiale. Cette période se caractérise par l'apparente consolidation de l'Empire français, malgré la montée du nationalisme anticolonial au Maroc et en Syrie, et la multiplication des révoltes anticoloniales, notamment au Kongo Wara en Afrique-Equatoriale française (AEF), à Yen Bay en Indochine (Thomas, 2007, p. 211).

Cette recherche se fonde sur la consultation de multiples sources: les archives nationales de l'outremer, les archives diplomatiques, les archives du Service historique de la Défense et les archives nationales de Pierrefitte, les archives nationales de Tunisie, la presse associative et la presse coloniale. Les archives coloniales ont été produites «par et pour les institutions qui dominent la population » (Surun et alii, 2012, p. 43) présente dans les colonies. Les sources consultées renseignent davantage sur les droits conférés aux mutilés que sur leurs expériences concrètes et leurs trajectoires de vie. Elles fournissent de nombreuses informations sur les territoires d'Afrique du Nord, mais ne donnent que des informations plus fragmentaires sur les autres territoires. Bien qu'elles soient très hétérogènes, ces sources reflètent généralement le point de vue de l'administration, et dévoilent rarement les revendications des mutilés. Dans ce cas, elles ne donnent accès qu'à la parole des mutilés européens, et presque jamais des mutilés indigènes, alors qu'ils sont majoritaires et les plus discriminés.

Nous étudierons dans un premier temps l'application des législations dans l'Empire colonial français, puis dans un second temps la résolution différenciée du problème de l'insertion professionnelle des mutilés selon le type de populations (indigènes/citoyens français), pour enfin terminer sur l'inégalité des ressources financières et ses conséquences sociales.

\section{L'application tardive, partielle et complexe des législations dans l'Empire colonial français}

L'adoption et l'application des législations d'assistance aux mutilés de guerre dans l'Empire colonial s'effectuent après d'intenses échanges épistolaires entre les gouverneurs locaux et les ministères de la métropole (Guerre, Colonies, Affaires étrangères, Travail, Pensions). Ces échanges contribuent à déterminer la nature des prestations délivrées et le périmètre des droits concédés, spécifiques à chaque territoire.

\section{1. Un retour tardif au pays? Une présence longtemps « indésirable » au Maroc.}


À partir de la fin de l'année 1914, certains mutilés de guerre français et indigènes appartenant aux corps réguliers de l'armée française retournent dans les pays d'Afrique du Nord après avoir été réformés. Mais dès janvier 1915, les autorités sont alarmées par le fait que des mutilés musulmans rapatriés propagent l'idée qu'ils ont été utilisés comme de la chair à canon devant les régiments français. Le comité interministériel sur les affaires musulmanes décide alors de suspendre le rapatriement des soldats mutilés vers l'Algérie et le Maroc (Thomas, 2005, p. 22). Les autorités sont aussi préoccupées par le fait que des soldats mutilés puissent se retrouver sans ressources dans leur pays d'origine, ce qui serait susceptible de donner l'image d'une France désintéressée par le sort des indigènes qui se sont sacrifiés pour elles $^{2}$; et ainsi d'alimenter la propagande anticoloniale. De ce fait, le ministre de la Guerre approuve rapidement les modalités d'attribution des allocations journalières provisoires aux militaires indigènes des corps réguliers (dépêche du 18 mars 1915) puis aux militaires indigènes des troupes auxiliaires algériennes et marocaines (décret du 28 juillet 1915).

Toutefois, le ministre de la Guerre s'oppose toujours au rapatriement généralisé des mutilés indigènes en instance de réforme, car la présence massive d'infirmes non rétablis, et/ou sans ressources, dans les dépôts militaires de Blida et de Casablanca, constituerait un «spectacle [qui] serait des plus fâcheux pour les recrues et la population $»^{3}$. De ce fait, les mutilés peuvent être envoyés «par petits groupes » dans ces dépôts, mais seulement après avoir été soignés et appareillés, et avoir obtenu leur réforme (permettant d'obtenir l'allocation journalière spéciale) et si besoin les décorations méritées. Comme l'indique le ministre de la Guerre Alexandre Millerand, ces mutilés des corps auxiliaires doivent être « entourés de tous les soins moraux et matériels et de toute la sollicitude reconnaissante qu'ils méritent ${ }^{4}$.

En mai 1915, 5 tirailleurs marocains réformés arrivent à Casablanca. Le Général Lyautey, Résident général à Rabat, refuse la généralisation de ces retours, du fait des inconvénients majeurs que ces retours pourraient avoir pour le recrutement des bataillons de tirailleurs marocains et pour la «politique indigène ${ }^{5}$. Leur présence serait susceptible d'alerter les populations locales sur les conséquences sociales de la guerre. Cette décision est validée par le ministre de la Guerre, ce qui contraint les mutilés marocains à séjourner au dépôt d'Arles. Certains mutilés semblent souffrir de cette situation, n'hésitant pas à commettre des tentatives de suicide (Bekraoui, 1987, p. 241).

À partir de 1916, la très grande majorité des militaires réformés sont renvoyés chez eux, exception faite de trois catégories d'entre eux : les militaires indigènes aliénés (internés dans un Asile d'Aix-en-Provence) ; les militaires tuberculeux (dirigés sur le centre de réforme de Marseille) ; les mutilés souhaitant suivre une rééducation professionnelle comme cordonnier ou tailleur dans la métropole ${ }^{6}$. À la fin de l'année 1920, la quasi-totalité des mutilés originaires des territoires de l'Empire a quitté la métropole. Une fois de retour dans

\footnotetext{
${ }^{2}$ Service Historique de la Défense (SHD), GR 7 NN9 1006. Lettre, Paris, le 25 mars 1915, Graziani, Général sous-chef d'État major de l'Armée au Commandant en chef des forces de terre et de mer de l'Afrique du Nord.

${ }^{3}$ SHD, GR 7 NN9 1006. Lettre, Paris, 6 mai 1915, le ministre de la Guerre au Général commandant la $15^{\mathrm{e}}$ région.

${ }^{4}$ SHD, GR 7 NN9 1006. Lettre, Paris, 14 août 1915, le ministre de la Guerre au Général Gouverneur Militaire de Paris.

5 SHD, GR 7 NN9 1006. Copie d'un télégramme, Casablanca, 19 mai 1915, Commissaire résident général au Ministère de la Guerre.

${ }^{6}$ SHD, GR 7 NN9 1006. Lettre, Paris, 13 avril 1916, le ministre de l'Intérieur au ministre de la Guerre.
} 
leurs pays, ils se font parfois les vecteurs de maladies contagieuses (tuberculose, maladies vénériennes, etc.) (Echenberg, 2009, p. 151).

\section{2. Des pressions politiques exercées par les associations des mutilés pour obtenir l'égalité des droits, relayées par l'ONMR et les parlementaires.}

Quatre acteurs contribuent à faire pression pour que des politiques d'assistance aux mutilés de guerre soient développées dans les territoires de l'Empire : les ministres de la Guerre et des pensions; les associations de mutilés des territoires de l'Empire et de la métropole, l'Office national des mutilés et réformés (ONMR) et certains parlementaires.

Au premier semestre 1916, les ministres de la Guerre et du Travail invitent les autres ministres (Colonies, Affaires étrangères) et les gouverneurs des colonies à organiser la rééducation professionnelle des mutilés de guerre dans les différents territoires de l'Empire. Le ministre du Travail et de la Prévoyance, président de l'Office National des Mutilés, enjoint alors le ministre de Colonies à favoriser la réinsertion professionnelle des mutilés de guerre, afin que ceux-ci puissent «reprendre leur place parmi les autres travailleurs ${ }^{7}$. Il recommande d'éviter la création de sociétés de secours mutuels propres aux mutilés, et de favoriser la création de comités locaux chargés de la défense des mutilés. Le ministre des Colonies obtempère rapidement. Dans plusieurs territoires de l'Empire, des sociétés de secours mutuels accueillent les mutilés de guerre et leur offrent de nombreuses prestations (frais d'hospitalisation, secours, etc.). Dans d'autres territoires (Algérie, Tunisie, Maroc, Madagascar), les mutilés créent leurs propres sociétés de secours mutuels.

Les mutilés de guerre constituent rapidement des associations de défense de leurs droits en Algérie (dès août 1915), au Maroc (1919), en Tunisie (1919), au Sénégal (1920), et à Madagascar (1926). Certaines de ces associations constituent des filiales des grandes fédérations nationales de mutilés et d'anciens combattants de la métropole. La grande majorité de ces associations sont des associations de secours mutuels qui versent des secours à leurs adhérents (prime de naissance, de décès, paiement des frais médicaux, etc.) et qui luttent pour conquérir des objectifs économiques et matériels : c'est le cas de l'Association générale des mutilés de la Grande Guerre en Tunisie, et de l'Amicale des mutilés et réformés de la Guerre de Madagascar. Certaines associations déploient d'autres activités, telle l'Amicale des mutilés de Constantine qui organise des cours d'alphabétisation. Preuve du dynamisme associatif dans les territoires d'Afrique du Nord, une interfédération nord-africaine des groupements des victimes de la guerre est créée en juin 1922. Dominée symboliquement par les mutilés, celleci rassemble 37400 adhérents en Algérie, 7200 au Maroc et 5000 en Tunisie en $1934^{8}$.

Ces associations rassemblent des Français et des Indigènes de toutes les classes sociales, mais celles d'ampleur nationale sont systématiquement dirigées par des Français. Prenons l'exemple de l'Association générale des mutilés de la Grande Guerre de Tunisie. Elle est présidée par René Soulmagnon, ancien avocat au barreau de Tunis, blessé au débarquement de Sed-ul-Bahr, entré dès 1916 à la Direction de l'Agriculture et du Commerce du gouvernement de Tunis. Il gravit les échelons jusqu'à devenir directeur de l'agriculture, du

\footnotetext{
7 Archives nationales de l'Outre-Mer (ANOM), 61COL 808. Lettre, Tananarive, le 1er juillet 1916, le Gouverneur délégué dans les fonctions de Gouverneur général de Madagascar au ministre des Colonies.

${ }^{8}$ Archives nationales, Pierrefitte (ANP), 20050299/45. ONAC, document intitulé «Groupements de victimes de la guerre et d'anciens combattants de l'Afrique du Nord, mars 1934 ».
} 
commerce et de la colonisation en 1934. Quelques mutilés indigènes (Rouidi Hadi, Mansour Ben Mohamed Ben Mansour) occupent néanmoins des postes subalternes (assesseurs indigènes) au sein du conseil d'administration ${ }^{9}$. Ces associations nationales obtiennent l'appui de nombreux médecins coloniaux, et ont des relations cordiales avec les gouverneurs locaux. Des associations locales sont dirigées par des musulmans, telle l'Union des mutilés et anciens combattants musulmans du Sud tunisien, qui se forme à Sfax en février $1938^{10}$.

Dans un contexte colonial répressif, ces associations ont un répertoire d'actions assez limité, se fondant sur des actions non violentes et non subversives : négociations politiques et administratives, pression auprès des parlementaires, interpellation publique des responsables politiques lors des congrès. Le recours aux négociations politiques et administratives est toutefois entravé par la distance géographique qui les sépare des ministères métropolitains qui concentrent le pouvoir de décisions. Pour y remédier, les responsables associatifs de certains territoires coloniaux (Algérie, Maroc, Tunisie) n'hésitent pas à se rendre à Paris pour négocier directement avec les fonctionnaires et responsables politiques. Ils invitent également les parlementaires et les ministres à venir à leurs congrès nationaux, ce qui leur offre la possibilité d'une négociation interpersonnelle à cette occasion. Lors de ces congrès, les associations déploient un argumentaire fondé sur l'égalité des droits de tous les mutilés compte tenu de leur sacrifice similaire pour la France, et la dénonciation des injustices liées à l'application partielle des législations métropolitaines. En 1924, le délégué des mutilés marocains déclare ainsi qu' «il faut traiter tous les mutilés sur un pied d'égalité - quelle que soit son origine, le sang versé pour la France a toujours la même valeur ${ }^{11}$. Toutefois, les critiques doivent se faire subtiles, et se fondre dans des preuves de loyauté vis-à-vis des autorités pour être audibles par ces dernières. En 1929, le président de l'interfédération nordafricaine des groupements des victimes de la guerre, Mr Soulmagnon, déclare :

"l'œuvre de réparation était trop formidable pour qu'elle pût se réaliser sans heurts, sans retard et sans erreurs individuelles (...). Les "créanciers sacrés de la Nation", ont terriblement souffert de ces vicissitudes et en pâtissent encore. Chez les meilleurs des nôtres, esprits loyaux et simples, la colère a grondé maintes fois, colère facile à exploiter. Eh bien, proclamons le aujourd'hui pour la première fois, c'est le plus grand honneur des groupements de victimes de la guerre de l'Afrique du Nord de n'avoir jamais cédé à cette colère, d'avoir placé au-dessus de tout le devoir national et d'avoir, par leur énergie pondérée, mais inlassable, fait rendre justice à leurs ressortissants en maintenant chez eux l'amour de la France $\gg^{12}$.

Les revendications des mutilés des colonies sont aussi relayées par l'Union fédérale, l'une des deux plus grosses fédérations nationales de mutilés, assez proches des radicaux socialistes. Leurs requêtes sont aussi appuyées par de nombreux parlementaires mutilés ou sympathisants de leur cause issus de plusieurs bords politiques, y compris de ceux favorables au maintien des hiérarchies coloniales : la droite libérale et républicaine (Marcel Ferraris,

\footnotetext{
${ }^{9}$ Bulletin trimestriel de l'Association générale des mutilés de la Grande Guerre en Tunisie, n 19 , avril 1923.

${ }^{10}$ Archives nationales de Tunisie (ANT). Série E, 509, dossier 278. Statuts de l'association "Union des mutilés et anciens combattants musulmans du sud tunisien', 1938.

${ }^{11}$ France Maroc, revue mensuelle illustrée, juin-juillet 1924, p. 104.

${ }^{12}$ ANT, Série E, 509, dossier 934. Article « Le VIIe congrès interfédéral des victimes de la Guerre », La Dépêche tunisienne, ler avril 1929.
} 
Maurice Kempf, etc.), les radicaux-socialistes (Henri Queuille) et les socialistes (Emile Jean Morinaud). En avril 1919, le député radical-socialiste Henri Queuille dépose une proposition de loi tendant à rendre applicable la loi du 2 janvier 1918 à l'Algérie, aux colonies et aux pays de protectorat. Cette proposition de loi n'aboutit jamais, mais elle pousse les ministères à conclure un projet de décret sur le sujet. De même, le député Georges Barthélemy alerte en avril 1922 le gouverneur général de l'AOF sur les injustices faites aux mutilés et l'absence de reconnaissance des autorités envers eux ${ }^{13}$.

Dès 1916, les dirigeants de l'ONMR font pression auprès des différents ministères (Colonies, Affaires étrangères) pour faire appliquer les lois dans les territoires de l'Empire (en particulier la loi du 2 janvier 1918). Cette pression de l'ONMR s'accentue en 1920, en raison de la participation d'un représentant algérien au sein de l'ONMR, et du changement de tutelle au profit du ministère des pensions. En effet, le ministre des Pensions André Maginot, ancien blessé de guerre, s'avère un allié puissant qui appuie les revendications des mutilés face au ministère des Finances jusqu'en 1924 (Prost, 1977). Les ministres des Pensions successifs continuent d'appuyer les revendications des mutilés, et ils multiplient les égards envers ceux de l'Empire à partir de 1929. C'est ainsi que par exemple que le ministre des Pensions se rend au congrès nord-africain des victimes de la guerre organisé à Tunis le 30 mars 1929. Des représentants associatifs mutilés de l'AOF (M. Baye et Joseph Memdy) et de l'Afrique du Nord sont invités respectivement en métropole en 1930 et 1931, pour les célébrations commémoratives de la Première Guerre mondiale ${ }^{14}$.

\section{3. Les ministères des Colonies et des Affaires étrangères confrontés à des choix complexes : l'impératif de la décentralisation.}

L'ONMR, le Ministère du Travail (jusqu'en septembre 1920) puis le Ministère des Pensions (après septembre 1920), ainsi que les associations de mutilés, sont favorables à l'application complète des législations dans les territoires de l'Empire. Ils se confrontent souvent à l'opposition du ministère des Finances, qui souhaite réduire au maximum les dépenses engendrées par ces mesures. Les ministères des Colonies et des Affaires étrangères restent très prudents sur les possibilités de leur application. Pour ces ministères, la décision d'appliquer ces dispositifs est d'autant plus complexe que se mêlent des questions administratives (dispositifs législatifs variables en fonction des territoires), des enjeux politiques (pression politique des associations, devoir de reconnaissance), des fondements statistiques incomplets (nombre de bénéficiaires) et des enjeux économiques (financement des institutions de rééducation, des pensions, de la gratuité des soins) et des enjeux sociaux et culturels (applicabilité à la société locale). Ils se trouvent donc confrontés à une situation d' « hyperchoix », «c'est-à-dire un choix qui relève de logiques fondamentalement différentes » (Jobert et Muller, 1987, p. 41). Ayant constaté les défaillances engendrées par la centralisation des politiques destinées aux mutilés de guerre dans la métropole, ces ministères veulent éviter cet écueil dans l'Empire. Pour fonder leur décision, ils sollicitent l'avis des gouverneurs sur les besoins locaux (utilité des écoles de rééducation professionnelle, des centres d'appareillage, etc.). Cette décentralisation de la décision est alors courante dans la gestion administrative de

\footnotetext{
${ }^{13}$ Les Annales coloniales, 18 avril 1922.

${ }^{14}$ Les Annales coloniales, 2 janvier 1930.
} 
l'Empire, du fait des distances, et «du nombre très limité d'administrateurs par rapport à l'immensité des territoires à administrer»(Dimier, 2003, p. 86). Les gouverneurs locaux disposent de pouvoirs étendus sur le plan réglementaire. Ils sont d'ailleurs libres d'exécuter ou non rapidement les textes métropolitains, car ils ne sont tenus à aucun délai dans la promulgation des décrets exécutoires (Dimier, 2003, p. 85).

En décembre 1917, le Président du Conseil, ministre de la Guerre, détermine la création de centres d'appareillage dans l'Empire ${ }^{15}$. Le ministère souhaite concentrer ces centres dans les capitales des principales zones de recrutement de l'Empire (Antilles, AOF, Indochine, Madagascar), tout en tenant compte du nombre de bénéficiaires potentiels. Quelques mois plus tard, après avis et accord des gouverneurs locaux, trois centres d'appareillage pour mutilés de guerre sont organisés à Hanoï, à Fort-de-France et à Dakar. À Madagascar, le gouverneur estime inutile de créer un centre d'appareillage pour les indigènes, considérant préférable que la métropole continue à fournir des appareils neufs aux mutilés. En 1918, un centre d'appareillage est aussi créé à Alger, afin de recevoir tous les mutilés d'Afrique du Nord. Par la suite, deux sous-centres sont organisés à Constantine et à Oran. Deux nouveaux centres sont ensuite constitués à Casablanca (Maroc) et à Salammbô (Tunisie), rattachés au centre d'appareillage de Bordeaux. Ces centres d'appareillage dispensent des dizaines de milliers de prothèses et d'orthèses aux mutilés. Ils semblent bien fonctionner, sauf à Dakar où la taille du centre semble insuffisante comparée aux besoins de toute l'AOF. Environ la moitié des prothèses distribuées dans le centre de Dakar proviennent de Bordeaux, les autres sont fabriquées sur place. En avril 1922, M. Adolphe Memdy, président du Groupe des mutilés de guerre de Dakar, réclame l'agrandissement du centre d'appareillage et de rééducation professionnelle, ainsi que le fait de « laisser au mutilé le libre choix de son appareil et de son orthopédiste ${ }^{16}$. Les mutilés de l'AOF éprouvent aussi des difficultés à faire réparer leurs prothèses du fait de l'éloignement vis-à-vis du centre (Echenberg, 2009, p. 254).

Lorsqu'elles envisagent l'application de la loi du 18 janvier 1918 aux colonies et aux territoires sous protectorat, les autorités appliquent la décentralisation des décisions, laissant aux gouverneurs locaux le soin de régler par arrêtés les détails de la mise en action de la loi (liberté de créer ou non un comité local relié à l'ONMR et un centre de rééducation professionnelle). Après avoir envisagé un temps d'organiser des écoles de rééducation à Hanoï et à Fort-de-France, le ministère des Colonies abandonne ces projets suite aux réponses négatives des gouverneurs locaux, invoquant le faible nombre de mutilés rééducables ${ }^{17}$. Le ministère du Travail et de la Prévoyance sociale approuve le 23 septembre 1919 deux décrets qui rendent applicable à l'Algérie la loi du 2 janvier 1918. Ces décrets prévoient la création de comités rattachés à l'ONMR, et la présence de membres indigènes aux côtés des membres européens nommés par le préfet au sein du comité départemental. De plus, ces décrets encouragent les comités - sans les contraindre - à créer des écoles de rééducation dans les départements qui en sont insuffisamment pourvus. L'adoption des mêmes dispositions dans

\footnotetext{
${ }^{15}$ ANOM, 61COL 808. Note, Paris, 30 novembre 1918, le ministre des Colonies au ministère du Travail et de la Prévoyance sociale.

${ }^{16}$ Les Annales coloniales, 18 avril 1922.

${ }^{17}$ ANOM, 61 COL 808. Note, 30 novembre 1918, le président du conseil supérieur de Santé des Colonies au directeur des services militaires.
} 
les pays sous protectorat est plus tardive : la résidence générale de Tunisie adopte le 26 mars 1922 un décret beylical appliquant la loi du 2 janvier 1918, sur le modèle des décrets algériens. Le Général Lyautey, résident général de la République française au Maroc, est au départ favorable à une application très limitée de la loi du 2 janvier $1918^{18}$. Les pressions répétées de l'ONMR et du Ministère des Affaires étrangères peinent à vaincre les réticences de la résidence générale au Maroc, qui remet en question en 1921 la nécessité d'adopter « un tel appareil réglementaire et législatif » étant donné l'action déjà réalisée dans le domaine de l'insertion professionnelle, et le faible nombre de bénéficiaires potentiels sur le plan de la rééducation $^{19}$. Elle finit par céder partiellement en 1924 en acceptant la création d'un Office du mutilé et du combattant.

Suite à l'adoption de ces dispositions, des comités de mutilés et réformés de la guerre sont constitués dans les départements algériens en 1920, en Tunisie et en Guadeloupe en 1922 et au Maroc en 1924. En Guadeloupe, le comité colonial des mutilés ne démarre réellement son action qu'en juin 1923, en aidant administrativement les mutilés à faire reconnaître leurs droits (pension, etc.), à trouver un apprentissage ou un emploi. Il agit peu jusqu'en 1925 pour des raisons administratives d'absence de validation du budget. L'action du comité est ensuite réorientée vers la distribution directe d'argent sous forme de prime de natalité, de secours au décès, de prêts aux mutilés et de subventions aux associations de victimes de la guerre ${ }^{20}$. Dans les autres territoires de l'Empire (AOF, AEF, Madagascar, Indochine, etc.), aucun comité de mutilés n'est créé, mais des comités coloniaux d'anciens combattants sont constitués après 1929.

Les caractéristiques des textes réglementaires attribuant des droits aux mutilés de guerre de l'Empire sont similaires à d'autres législations sociales s'appliquant dans les territoires de l'Empire : ils prévoient une application partielle et tardive des dispositions métropolitaines, avec des différentiations selon les territoires et les populations (français/indigènes) (Lekeal, 2014). Deux spécificités méritent d'être soulignées : premièrement, du fait de leur caractère non contraignant, certaines législations (notamment celles dupliquant la loi du 2 janvier 1918) jouent un faible rôle pour faire avancer les politiques en faveur des mutilés de guerre. Avant même l'adoption de ces législations, les gouverneurs locaux et les associations entreprennent de nombreuses actions en faveur des mutilés de guerre (création de centres de rééducation professionnelle, etc.). D’une part, dans plusieurs colonies (Madagascar, Guyane, Tahiti), des comités d'aide aux soldats réformés sont organisés très tôt, dès 1916, présidés par des maires des capitales des colonies ou des notables locaux. D'autre part, l'application de la loi du 2 janvier 1918 n'entraîne pas la réouverture d'écoles de rééducation dans les territoires d'Afrique du Nord, au grand dam des mutilés concernés. Deuxièmement, alors que les gouverneurs locaux semblent développer une grande diversité de politiques sociales destinées à minimiser les obligations de l'État envers les pupilles de l'Empire (Hassett, 2016, p. 340), les veuves de guerre, et les aveugles civils, ils n'adoptent pas systématiquement cette attitude envers les mutilés de guerre. Ces derniers

\footnotetext{
${ }^{18}$ Centre des archives diplomatiques de la Courneuve, (CADC), K - Afrique, 53CPCOM/013740. Lettre, 30 octobre 1920, le prsdt du comité d'administration de l'ONMR au prsdt du Conseil, MAE.

${ }^{19}$ CADC, K - Afrique, 53CPCOM/013740. Lettre, 1 avril 1921, le Délégué à la résidence générale de la RF au Maroc au Prsdt du Conseil, MAE.

${ }^{20}$ ANOM, 61 COL 808. Rapport, Basse Terre, 9 décembre 1926, le gouverneur au prsdt de l'ONMR.
} 
accèdent plus facilement à des bénéfices économiques conséquents, notamment sur le plan des pensions et de l'emploi, du fait de leur sacrifice pour la "Patrie" et de l'impossibilité de les discriminer trop fortement sans risques politiques.

\section{Des offres importantes d'emplois pour les mutilés européens, en moindre nombre pour les mutilés indigènes.}

Selon les territoires de l'Empire, les gouverneurs locaux appliquent de manière variable les circulaires administratives envoyées par les ministres de la métropole, qui les enjoignent à organiser la réinsertion professionnelle des mutilés de guerre.

\section{1. Un placement rapide des mutilés français aptes à occuper un poste dans les administrations locales et quelques entreprises privées.}

Les administrations coloniales favorisent la réinsertion professionnelle des mutilés qui sont aptes à occuper un poste de travail, en leur réservant une partie des places vacantes dans les administrations coloniales au cours des années postérieures à la guerre. La plupart des gouverneurs locaux adoptent des dispositions juridiques en ce sens: le gouverneur de Madagascar adopte un arrêté en 1916 favorisant l'accès des mutilés français dans l'Administration locale, en accordant des facilités d'examens et en réservant des emplois peu qualifiés dans les communes (gardiens de cimetière, gardiens des eaux, etc.). En Tunisie, le gouvernement beylical adopte un décret (23 novembre 1919) réservant certains emplois subalternes pour les mutilés dans les administrations publiques. Toutefois, en AfriqueOccidentale française, les mutilés ne semblent pas bénéficier de mesures spécifiques en sus des mesures attribuées aux anciens militaires. En 1922, les mutilés de Dakar réclament ainsi au gouverneur général de l'AOF «que les emplois réservés aux anciens militaires leur soient accordés de préférence ${ }^{21}$.

L'intensité de l'application de ces textes juridiques est variable d'un territoire à l'autre. En Tunisie, moins d'une centaine d'emplois sont attribués à des mutilés au sein des administrations tunisiennes (gendarmerie, administration communale, etc.) jusqu'en $1924^{22}$. Les principaux bénéficiaires sont les mutilés français ayant des qualifications ou des diplômes. À Saint-Pierre et Miquelon, en 1919, « Sur 12 mutilés, 6 ont repris leurs anciennes professions, et les 6 autres ont reçu des emplois dans les divers services de la Colonie ${ }^{23}$. La Résidence générale au Maroc déploie une activité intense de placement au cours de la période 1917-1921. Sur 1360 mutilés rentrés de France, 1200 sont placés avant 1921 dans les administrations et les entreprises privées ${ }^{24}$. Le général Lyautey a visiblement la volonté de favoriser leur réinsertion professionnelle afin de les constituer en soutien politique de l'ordre colonial, dans un contexte politique tendu du fait de la dissidence berbère dans les montagnes du Rif, qui n'avait pas pris fin avec la guerre (Ganiage, 1994, p. 432). En Algérie, les administrations placent au moins plusieurs centaines de mutilés en leur sein, mais les

\footnotetext{
${ }^{21}$ Les Annales coloniales, 18 avril 1922.

${ }^{22}$ ANT, SG 6 - 135, dossier 8. Tableaux sur les emplois offerts aux mutilés, juin 1924.

${ }^{23}$ ANOM, 61 COL 808. E Colin, Note pour le Cabinet du ministre, 17 avril 1919.

${ }^{24}$ CADC, K - Afrique, 53CPCOM/013740. Lettre, 1er avril 1921, le Délégué à la résidence général au Maroc au MAE.
} 
associations se plaignent de l'absence d'une disposition juridique régulant l'emploi obligatoire des mutilés dans les entreprises privées à l'instar de la loi du 29 août $1924^{25}$.

Après 1922, les administrations coloniales se montrent moins soucieuses d'accorder la priorité de l'emploi aux mutilés de guerre, et privilégient parfois des "valides" temporairement. Les associations de mutilés d'Afrique du Nord contestent ces pratiques et exigent la réserve effective des postes vacants pour les mutilés ${ }^{26}$. Suite à la parution d'un nouveau décret le 17 juillet 1929 dans la métropole, une nouvelle procédure d'emplois réservés pour les mutilés et anciens combattants est lancée au début des années 1930 dans les territoires d'Afrique du Nord. Officialisée par des décrets pris à l'échelle de chaque territoire de l'Empire, cette nouvelle procédure conduit à l'embauche de nombreux mutilés les premières années, mais l'administration tunisienne la contourne très rapidement en déclassant les emplois réservés en postes d'auxiliaires, afin que leur attribution soit décidée par le secrétariat général du gouvernement et non par la commission sur les emplois réservés où sont représentées les associations de mutilés. Cette situation indigne l'Association générale des mutilés de la Grande Guerre en Tunisie, qui proteste contre ces déclassements et réclame la réservation des postes d'auxiliaires pour les mutilés et victimes de la guerre ${ }^{27}$.

Toutefois, l'administration coloniale n'est pas la seule à agir pour le placement des mutilés : les associations de mutilés, les comités de mutilés et certains services municipaux y travaillent activement.

\section{2. Le douloureux problème des mutilés impossibles à placer dans l'emploi.}

En fonction de son infirmité, l'individu ne peut concourir qu'à une liste prédéfinie d'emplois déterminés sur le plan législatif. Ainsi, l'ex-soldat Léon Colin voit sa candidature pour l'emploi de «porteur de contraintes ${ }^{28}$ en Algérie rejetée pour «Aptitude physique insuffisante $» d u$ fait de son infirmité (résection du coude droit, ankylose) ${ }^{29}$. Il ne peut postuler qu'à deux emplois : facteur et concierge des tribunaux. Les mutilés ayant des infirmités sévères, ou des infirmités jugées incompatibles avec la plupart des emplois (trépanés, sourds, etc.) ont des possibilités plus restreintes de trouver un emploi.

Du fait de leur statut administratif spécifique, les mutilés indigènes bénéficient d'un nombre très restreint d'emplois réservés dans les administrations. En 1924, seuls 12 mutilés indigènes occupent un poste au sein des administrations tunisiennes, dont 7 comme Cheikhs ${ }^{30}$ et 3 comme Amines ${ }^{31}$.

La majorité des mutilés indigènes sont d'anciens ouvriers agricoles, mais leur infirmité complique généralement la reprise d'une activité agricole. Faute de qualifications, les mutilés

\footnotetext{
${ }^{25}$ L'Algérie mutilée, 16 avril 1928.

${ }^{26}$ Le mutilé de l'Algérie, Alger, 11 février 1923, p. 15.

27 ANT, SG6-257, dossier 3. Congrès fédéral des mutilés, AC et victimes de la guerre. Vøux, Congrès du 31 mars 1935.

${ }^{28}$ Personne notifiant au contribuable les mises en demeure de payer du percepteur.

${ }^{29}$ Bulletin Mensuel Les Mutilés, Oran, n60, octobre 1922, p. 16.

${ }^{30}$ Personnes chargées de la supervision d'une petite division administrative.

${ }^{31}$ Personnes représentant une corporation, chargées de la vérification des poids et mesures, et des conflits entre patrons et ouvriers.
} 
indigènes peinent à être embauchés dans les entreprises ou l'industrie. Dans les territoires d'Afrique du Nord, des emplois spécifiques dans le domaine du commerce leur sont réservés : en Algérie, ils bénéficient de la majorité du contingent autorisé des exportations d'oeufs, tout comme des autorisations d'ouverture de débits de cafés maures. Toutefois, les irrégularités observées lors des exportations d'œufs entraînent le retrait définitif de ces licences d'exportation. De plus, un nombre restreint d'indigènes ont la possibilité de détenir un café maure, en l'absence de places vacantes : au premier semestre 1926 dans le département d'Oran, seules deux places de cafetier maure sont attribuées à des mutilés indigènes, pour 522 demandes $^{32}$. Au Maroc, 91\% des débits de tabac sont affectés à des mutilés (Français ou Indigènes) ou à des veuves. Afin de trouver de nouveaux débouchés professionnels pour les indigènes, les associations réclament aussi la réserve des postes de chaouchs (appariteurs des services publics) ou de spahis (cavalier) ${ }^{33}$.

Dans chaque territoire où il existe une forte proportion de mutilés de guerre (Afrique du Nord, AOF), plusieurs centaines d'entre eux souhaitant travailler restent sans activité rémunérée pendant de nombreuses années. Beaucoup de mutilés indigènes « chôment malgré eux ${ }^{34}$ en raison de l'insuffisance des politiques développées par les gouvernements locaux. Leur situation économique s'aggrave au cours des années 1930, avec la crise économique et la baisse de la valeur relative des pensions.

\section{3. L'échec partiel de la rééducation professionnelle des mutilés illettrés.}

Dès 1916, des ateliers de cordonniers et de tailleurs sont organisés dans les dépôts de Tirailleurs à Casablanca et à Blida. De véritables centres de rééducation professionnelle sont organisés la même année dans les départements algériens (à Kouba, Alger, Oran), et les années suivantes dans les pays sous protectorat (La Goulette près de Tunis, Casablanca) et à Dakar (alors chef-lieu de l'AOF). Le département d'Alger finance la création d'un centre de rééducation à Kouba (qui accueille en tout 1437 élèves) ${ }^{35}$, et celui d'Oran subventionne la création de l'Ecole Victor Vassal à Oran (qui accueille au moins 300 élèves en tout), conjointement avec l'Union des femmes de France. Les services de santé militaires des gouvernements participent à l'organisation de plusieurs centres de rééducation (Kouba, Casablanca, La Goulette). Ces institutions dispensent généralement des enseignements manuels : vannerie, fabrication de nattes, sparterie, brosserie, menuiserie, forge. À l'instar de nombreuses institutions de la métropole, l'objectif est d'en faire de petits artisans indépendants (Collard, 2018). De manière plus marginale, l'association d'assistance aux mutilés de Salammbô organise une rééducation professionnelle dans le domaine de l'agriculture, et l'Amicale des Mutilés du Département d'Alger organise un cours d'enseignement général. Si les institutions algériennes sont destinées aux mutilés européens et indigènes, celui de Casablanca est explicitement destiné aux anciens tirailleurs indigènes sans instruction.

\footnotetext{
${ }^{32}$ Bulletin Mensuel Les Mutilés, Oran, n¹06, août 1926, p. 3.

${ }^{33}$ Le Mutilé de l'Algérie. 11 février 1923, p. 15.

${ }^{34}$ Ibid., p. 7.

${ }^{35}$ L'Algérie mutilée, 1er juin 1923.
} 
Cette politique de rééducation professionnelle s'avère un échec partiel. Le nombre de bénéficiaires n'est pas négligeable (au moins deux mille dans l'Empire) mais ne couvre pas la totalité des besoins du fait de la fermeture rapide des écoles de Casablanca, Oran et de Tunis dès 1920, et de Kouba en 1922. Ces formations connaissent parfois des difficultés de fonctionnement (problème de direction, gaspillage de matières premières, etc.) et n'obtiennent pas les résultats escomptés : les mutilés rééduqués à trouver un emploi à la sortie sont peu nombreux, notamment en raison de l'impossibilité de constituer une coopérative de production. Au Maroc, les autorités sont enclines, par racisme, à considérer que les mutilés indigènes sont fainéants par nature et peu motivés dans leur apprentissage. Le délégué à la résidence générale de la France au Maroc considère avec mépris que les mutilés du Centre de Casablanca «ne voulaient rien apprendre; et cela très certainement parce qu'ils constituaient le déchet des 1360 Marocains réformés [dont 1200 étaient déjà placés] » ${ }^{36}$. Les mutilés en ont une interprétation différente, considérant que les résultats médiocres du centre «sont dus en grande partie à une direction défectueuse $»^{37}$.

Au début des années 1920, les gouverneurs du Maroc, d'Algérie et de Tunisie considèrent que l'échec des essais de rééducation professionnelle passés rend illusoire tout nouvel essai. Les revendications émises par les mutilés en faveur de la réouverture d'une école au Maroc, ou de l'organisation de cours (comptabilité, dactylographie) ou d'apprentissage chez les patrons (menuiserie, cordonnerie, bourrellerie) en Algérie et en Tunisie ${ }^{38}$, restent lettres mortes.

Le centre de rééducation de Dakar subsiste plus longtemps que les autres, mais il reste d'une taille insuffisante face aux besoins. Le président du Groupe des mutilés de guerre de Dakar réclame ainsi en avril 1922 de «créer des ateliers de menuiserie de forge, de cordonnerie, de bourrellerie, de reliure, et de commencer des cours de comptabilité et de dessin industriel $»^{39}$. Nous ignorons si cette requête aboutit.

\section{4. Faciliter l'acquisition de terrains agricoles.}

Dans la métropole, les autorités favorisent la reprise d'une activité agricole en accordant aux mutilés l'acquisition de lots de terre, par le biais de prêts à visée agricole du Crédit Mutuel à un taux préférentiel (à $1 \%$, avec une bonification de l'État par enfant) (loi du 5 août 1920). La loi du 5 août 1920 n'est pas applicable aux territoires de l'Empire, mais les associations d'Afrique du Nord réclament l'application de cette loi et la priorité dans l'attribution des concessions de terres domaniales ${ }^{40}$. Suite à cette pression associative, les résidents généraux de la Tunisie et du Maroc leur accordent une priorité lors de l'attribution des lots de colonisation ou de terres domaniales. Au Maroc, en 1919, 25\% des lots de colonisation de taille moyenne sont réservés aux mutilés français. La résidence générale adopte le 27 décembre 1919 un dahir qui attribue aux anciens combattants une ou plusieurs

\footnotetext{
${ }^{36}$ CADC, K - Afrique, 53 CPCOM/013740. Lettre, 1er avril 1921, le Délégué à la RG de la RF au Maroc au Prsdt du Conseil, MAE.

${ }^{37}$ Le mutilé de l'Algérie, 11 février 1923, p. 19.

${ }^{38}$ Ibid.

${ }^{39}$ Les Annales coloniales, 18 avril 1922.

${ }^{40}$ Les terres domaniales sont des terrains qui appartiennent à l'État.
} 
parcelles de terre domaniale, d'abord en jouissance provisoire puis en pleine propriété. En moins de deux ans, plus d'une cinquantaine de mutilés bénéficient d'un lot de terre domaniale grâce à cette disposition ${ }^{41}$. En Tunisie, le protectorat réserve aux mutilés de guerre des petits lots de colonisation ( 60 hectares de terre cultivable), avec des prêts avantageux (12 000 francs à 2\%). Au moins 35 mutilés français bénéficient de cette disposition avant 1923.Une trentaine d'autres mutilés bénéficient d'un accès privilégié à des lots normaux de colonisation (100 hectares). Plusieurs dizaines de mutilés indigènes accèdent aussi à des terres attribuées par le protectorat aux agriculteurs indigènes au cours des années $1920^{42}$. À la différence des gouvernements tunisiens et marocains, celui de l'Algérie refuse de réserver aux mutilés des terres de colonisation, ayant la volonté de favoriser l'installation dans la colonie «des agriculteurs français $»^{43}$. Il se contente d'assouplir les règles de crédits à visée agricole pour les mutilés, ce qui permet à plus d'une centaine d'entre eux d'en obtenir au cours des années 1920.

Dans les autres territoires de l'Empire, nous n'avons pas retrouvé la trace de dispositions similaires favorisant l'installation des mutilés dans l'agriculture. En Guyane, Joseph Photius, mutilé de guerre habitant Cayenne, signale «l'injustice et l'injure faites aux blessés des Colonies notamment en Guyane ${ }^{44}$ du fait de l'absence d'application de la loi du 5 août 1920 , et demande à bénéficier d'un prêt pour accéder à une propriété rurale. Même dans les territoires où les autorités ne facilitent pas l'acquisition de terres agricoles, de nombreux mutilés contribuent aux activités agricoles «pour leur compte ou pour celui d'autrui », comme en Guadeloupe ${ }^{45}$.

Les multiples dispositifs d'insertion professionnelle mis en place pour les mutilés sont variables d'un pays à l'autre, les pays d'Afrique du Nord ayant une action plus importante dans ce domaine en raison de la forte pression associative. Le travail de placement dans l'emploi est destiné en priorité aux mutilés de guerre français. Cette attitude discriminatoire s'explique à la fois par les souhaits des gouverneurs locaux de respecter les hiérarchies coloniales (priorité aux mutilés français dans l'emploi), par les préjugés récurrents des administrateurs coloniaux, et par le faible niveau scolaire de ces mutilés, qui les rend peu aptes à occuper des postes administratifs.

\section{Des ressources financières suffisantes grâce aux pensions ?}

Une série de dispositions sont adoptées pour conférer à la grande majorité des mutilés de l'Empire certains bénéfices économiques non négligeables. La pension d'invalidité constitue le bénéfice économique le plus important de tous, sans être pour autant l'unique

\footnotetext{
${ }^{41}$ CADC, K - Afrique, 53CPCOM/013740, Lettre, 1 avril 1921, le Délégué à la résidence général de la RF au Maroc au Prsdt du Conseil, MAE.

${ }^{42}$ Bulletin Mensuel de l'Office du Protectorat français en Tunisie, février 1926, n 178, p. 28.

${ }^{43}$ Bulletin Mensuel Les Mutilés, Oran, n93, juillet 1925, p. 7.

${ }^{44}$ ANOM, 4106 COL76. Copie d'une lettre, Joseph Photius, Cayenne, 8 septembre 1927, à l'UNMR.

${ }^{45}$ ANOM, 61 COL 808. Lettre, Saint Claude, 4 septembre 1924, le gouverneur de la Guadeloupe et dépendances au ministre des Colonies.
} 
bénéfice concédé. En Algérie et en Tunisie, les mutilés disposent aussi de la gratuité des soins à partir de 1922, suite à une intense mobilisation associative. Dans les territoires d'Afrique du Nord et en AOF, les mutilés de guerre indigènes et européens ayant plus de 50\% d'invalidité bénéficient aussi de l'exemption de certains impôts (soit de la contribution personnelle mobilière, soit de l'impôt de capitation). Dans chaque territoire, les gouverneurs locaux offrent aussi à plusieurs dizaines de grands invalides un hébergement en institution (sanatoriums, camp militaire d'invalides en Côte d'Ivoire, Maison des invalides marocains de Mogador au Maroc, Centre d'hébergement de Kouba en Algérie à partir de 1922) ou leur attribuent un accès prioritaire à des logements en Habitations bon marché (en Tunisie).

La pension d'invalidité constitue une source de revenus importante et stable pour les mutilés (Surun, 2012, p. 194), sans toutefois toujours être leur unique ressource financière (revenus liés à l'emploi, à l'activité artisanale). Le système des pensions militaires constitue la principale mesure d'aide consentie par l'État français aux mutilés, aux anciens combattants et aux ayants droit des victimes de la guerre, la plus coûteuse de toutes (21 milliards de francs en 1920, et 50 milliards en 1934). Ces pensions engendrent un énorme transfert monétaire entre la métropole et les territoires de l'Empire, qui rejaillit ensuite sur la vitalité économique locale. Les autorités françaises cherchent à définir leur montant en se fondant sur quatre critères : le coût pour les dépenses de l'État français ; la nécessité de préserver les hiérarchies coloniales; les services rendus par les différents corps d'armée pendant la guerre; et les conséquences politiques du possible caractère inégalitaire des pensions entre français et indigènes (risque du renforcement de la lutte anticoloniale). Enfin, l'application du système des pensions pose des enjeux sociaux et économiques importants dans les territoires de l'Empire du fait de la polygamie de nombreux musulmans, alors que le système métropolitain de réversion des pensions aux veuves et aux orphelins est pensé pour des unions monogames.

La question des pensions d'invalidité ne peut pas être dissociée de celle des pensions par ancienneté. À partir de 1924, les autorités rompent la logique d'égalité des pensions d'ancienneté entre militaires français et indigènes nord-africains, en suspendant les augmentations pour les militaires indigènes, contrairement à celles des militaires français (loi du 14 avril 1924 et décret d'application du 10 décembre 1925). Le décrochage est déjà assez important en 1929 (un indigène touchant $75 \%$ et 85\% de la pension d'un français de même statut), et il s'aggrave du fait de l'augmentation du montant des pensions accordées aux militaires français au cours des années 1930, passant à environ 55 à $65 \%$ de la pension de retraite accordée aux Français en 1937. Ce décrochage suscite de nombreux débats en Afrique du Nord, ce qui alerte l'État major des Armées, qui s'avère inquiet « de ne pas laisser naître dans l'esprit des indigènes l'appréhension d'une déconsidération progressive de leurs services ${ }^{46}$. La différenciation des pensions par ancienneté pour les Français et les Indigènes crée un précédent important qui rejaillit ensuite sur la gestion des pensions d'invalidité.

Jusqu'au début des années 1920, les mutilés ne bénéficient pas de leur pension d'invalidité, mais d'une allocation provisoire d'attente d'un montant dérisoire, fixée d'après l'évaluation médicale de leur degré d'invalidité. Les médecins se fondent sur un guide barème indicatif, qui se révèle impératif pour les mutilations, la cécité et la tuberculose. Il est

\footnotetext{
${ }^{46}$ SHD, GR 7 NN 9 1037. Copie d'une lettre, non datée, le ministre de la Défense nationale et de la guerre au ministre des Finances.
} 
demandé d'appliquer le taux le plus favorable possible. Les mutilés ont la possibilité de faire valoir leur droit à pension dans les cinq ans qui suivent leur réforme pour invalidité. Des facilités sont proposées aux mutilés illettrés pour solliciter des pensions, notamment par le recours au système des empreintes digitales en $\mathrm{AOF}^{47}$. La grande majorité des mutilés de guerre qui en font la demande perçoivent une pension, qui se révèle d'un montant fort variable d'un territoire de l'Empire à l'autre. Au 31 décembre 1926, en AOF, 1057 mutilés européens et 10151 mutilés indigènes bénéficient d'une pension d'invalidité ${ }^{48}$. Nous ignorons le nombre de bénéficiaires des pensions d'invalidité dans les autres territoires de l'Empire.

\section{1. Des droits égaux à la pension pour les mutilés européens et indigènes en Afrique du Nord et dans les 4 communes du Sénégal, grâce à la loi du 31 mars 1919.}

La loi du 31 mars 1919 instaure un nouveau système de pensions pour les armées de terre et de mer dans la métropole et les territoires de l'Empire. Le montant de la pension pour invalidité varie en fonction du degré d'invalidité, du statut de chaque soldat vis-à-vis de l'armée française (corps régulier ou corps auxiliaires), et de son rang militaire. L'article 73 de cette loi détermine l'égalité des tarifs de pensions entre les militaires français et les «militaires indigènes de l'Algérie et des colonies ou pays de protectorat dans lesquels le recrutement s'opère par voie de conscription ${ }^{49}$. Le bénéfice de cette égalité de traitement ne concerne au départ que les indigènes recrutés en Algérie, en Tunisie et dans les quatre communes du Sénégal $^{50}$. Il est élargi ensuite aux indigènes marocains en 1924. Ce traitement différentiel entre indigènes de l'Empire, souhaité par le ministère de la Guerre, se fonde sur leur mode de vie : dans les pays d'Afrique du Nord, leur mode de vie est proche des Français, et «il n'en est pas de même dans nos autres colonies » ${ }^{51}$, notamment en AOF et en AEF où «les indigènes de race nègre ont conservé une organisation sociale qui ne saurait être comparée à la nôtre et leur genre de vie est loin de leur imposer les mêmes charges qu'aux citoyens français $»^{52}$. Ce traitement différentiel a deux objectifs, l'un économique, limiter les dépenses de l'État, et l'autre politique, se concilier la sympathie des populations indigènes de l'Afrique du Nord et du Sénégal. Par contre, l'article 74 de la loi du 31 mars 1919 différencie le système de réversion des pensions appliqué dans la métropole, et celui appliqué pour les ayants droit des militaires indigènes musulmans originaires d'Afrique du Nord et non naturalisés. Pour ces derniers, la pension est divisée en un nombre considérable d'ayants droit, sans possibilité de réversion entre les différents lits. Preuve des égards des autorités françaises pour les mutilés de ces territoires, le règlement d'administration publique portant application de la loi du 31 mars 1919 aux colonies est publié dès le 2 octobre $1919^{53}$.

Tableau $n^{\circ} 1$. Tableau des pensions maximales attribuées aux mutilés français et indigènes concernés par la loi du 19 mars 1919 (Algérie, Tunisie, quatre communes du Sénégal, puis Maroc).

\footnotetext{
${ }^{47}$ ANOM, 61 COL 192. Dépêche télégraphique, Dakar, 27 mars 1917, Clozel à Min. Colonies Paris.

${ }_{48}$ ANOM, 61 COL 192. Lettre, Dakar, 13 avril 1927, Le gouverneur général de l'AOF au ministre des Colonies.

${ }^{49}$ Journal Officiel de la République française, 2 avril 1919, p. 3389.

${ }^{50}$ SHD, GR 7 NN 9 1037. Note, Paris, 17 mars 1918, pour l'État major de l'Armée.

${ }^{51}$ SHD, GR 7 NN 9 1037. Brouillon d'une note, 28 fév 1918, Alby pour le service général des pensions.

${ }^{52}$ Ibid.

${ }^{53}$ Journal Officiel de la République française, 7 novembre 1919, p. 12491-12492.
} 


\begin{tabular}{|l|l|l|l|l|}
\hline & $10 \%$ & $50 \%$ & $80 \%$ & $100 \%$ \\
\hline Capitaine & 440 & 2200 & 3520 & 4400 \\
\hline $\begin{array}{l}\text { Adjudant- } \\
\text { chef }\end{array}$ & 260 & 1300 & 2080 & 2600 \\
\hline Soldat & 240 & 1200 & 1920 & 2400 \\
\hline
\end{tabular}

Source : JORF, 2 avril 1919, p. 8891.

Le montant de ces pensions augmente au cours des années 1920, grâce aux luttes menées par les associations de mutilés dans la métropole, qui obtiennent des augmentations en 1925 et 1927 afin de compenser l'inflation. Toutefois, il faut souligner que les mutilés indigènes de l'Afrique du Nord et des quatre communes du Sénégal perçoivent généralement des pensions d'un montant largement inférieur à celles des mutilés français des mêmes territoires: premièrement, du fait de leur maintien à des postes très bas dans la hiérarchie militaire (Fogarty, 2008 : 111) ; deuxièmement, du fait de leur appartenance à un corps militaire distinct de l'armée régulière française pendant un laps de temps plus ou moins long. Enfin, le système de réversion des pensions est très défavorable pour les ayants droit.

Du fait de leur appartenance aux corps auxiliaires, les tirailleurs algériens et les auxiliaires marocains ne bénéficient pas des droits accordés aux mutilés de l'armée régulière française jusqu'en 1919 pour les Algériens, et jusqu'en 1924 pour les Marocains. Le décret de juillet 1915 leur confère des allocations journalières provisoires minimales : les spahis algériens touchent une allocation égale à celle des militaires français et indigènes de $2^{\mathrm{e}}$ classe des corps réguliers, soit $1 \mathrm{fr} 70$; et les auxiliaires marocains bénéficient d'un taux variable en fonction du grade, de 1 fr 25 à 1 fr 70 pour les sous-officiers.

Si le cas algérien est réglé par la loi du 31 mars 1919, ce n'est pas le cas du cas marocain. Dès la période de la guerre, les mutilés indigènes marocains contestent cette inégalité de statut avec les mutilés français. Ils font pression sur les ministres de la métropole pour obtenir l'intégration des unités marocaines dans l'armée régulière française. Si les ministres de la Guerre et des Affaires étrangères sont favorables à cette intégration dans l'armée régulière française, le général Lyautey y est catégoriquement opposé, et il est soutenu par le ministre des Finances (Wanaïm, 2009). Les combattants indigènes du Maroc se voient refuser le bénéfice des pensions prévues par la loi du 31 mars 1919 du fait de leur appartenance aux troupes auxiliaires marocaines. La situation se débloque en janvier 1924, lorsque le ministre de la Guerre émet une dépêche pour accorder un nouveau titre de gratification aux anciens militaires des troupes marocaines ${ }^{54}$.

De plus, lorsqu'elles sont confrontées à des contraintes budgétaires, les administrations coloniales n'hésitent pas à discriminer les mutilés indigènes des territoires d'Afrique du Nord. En mars 1923, le ministère adopte ainsi une circulaire déniant le bénéfice des majorations pour enfants aux mutilés indigènes d'Algérie, ce qui révolte les associations de mutilés ${ }^{55}$. Saisi de la question par les associations, le Conseil d'État confirme finalement les mutilés indigènes algériens dans leurs droits concédés par la loi du 31 mars 1919.

\footnotetext{
${ }^{54}$ France Maroc, revue mensuelle illustrée, juin-juillet 1924, p. 104.

${ }^{55}$ La France mutilée, 3 juin 1923, p. 1.
} 
En 1928, l'État major de l'Armée se déclare favorable à la baisse des pensions d'invalidité pour les mutilés indigènes des pays d'Afrique du Nord, car les tarifs actuels «sont beaucoup trop élevés étant donné les conditions de la vie indigène », et "peuvent porter l'homme [...] à aggraver son infirmité et à ne rien faire pour hâter sa guérison ou améliorer son état de santé ${ }^{56}$. En prenant modèle sur la situation des pensionnés par ancienneté, il recommande au secrétariat général du Ministère de la guerre d'adopter " pour l'avenir, à l'égard des indigènes nord-africains, des tarifs de pension d'invalidité inférieurs à ceux des Français $»^{57}$. En raison des répercussions politiques d'une telle modification, les avis du gouverneur général de l'Algérie, du Résident général au Maroc et en Tunisie sont recueillis sur le montant des pensions d'ancienneté et d'invalidité. Les résidents généraux du Maroc et de Tunisie acceptent l'idée d'un décrochage des pensions des indigènes (maintien du même taux de pensions pour les indigènes en cas d'augmentation du montant des pensions des militaires français $)^{58}$. À l'inverse, le gouverneur général de l'Algérie considère indispensable de maintenir «la pleine égalité entre Français et indigènes », en raison de l'enjeu fondamental de la collaboration franco-indigène. Le Président du Conseil partage son avis. Informés de cette enquête, les mutilés d'Afrique du Nord réunis à Oran en 1930 protestent énergiquement contre cette possible révision et s'engagent à lutter pour conserver l'égalité des droits ${ }^{59}$. Cette pression associative, cumulée au positionnement du gouverneur de l'Algérie, et à l'absence d'augmentation du montant des pensions pour les mutilés français, contribue au maintien du statu quo en 1931. Le secrétariat général considère qu' «il n’y avait pas lieu de soulever la question pour éviter des polémiques inutiles ${ }^{60}$.

Tout au long des années 1930, les associations de mutilés d'Afrique du Nord réclament l'augmentation du niveau des pensions d'invalidité et d'ancienneté, mais nous manquons de sources pour dire si des augmentations sont accordées par les autorités.

\section{2. Des pensions d'un montant largement inférieur pour les mutilés indigènes des autres colonies (AOF, AEF, Madagascar, etc.).}

Les modalités de fixation des pensions d'invalidité des indigènes des autres colonies ne sont fixées qu'un an après la loi du 31 mars 1919, par le décret du 22 septembre 1920. Ce décret détermine des tarifs de pensions variables selon les colonies, en distinguant l'AOF et l'AEF (entre 40 et $60 \%$ du tarif attribué aux mutilés français suivant le grade), puis les autres colonies (Indochine, Côte des Somalis, Nouvelle-Calédonie, Océanie, touchant entre 30 et 43 $\%$ du taux des mutilés français), et enfin Madagascar (entre 25 et 32\% du tarif attribué aux mutilés français). Non seulement ces pensions sont très faibles, mais les grands invalides indigènes de ces territoires ne bénéficient pas des suppléments de pension concédés aux grands invalides bénéficiaires de la loi du 31 mars 1919 (majoration de $25 \%$ pour assistance à la tierce personne, et compléments en cas d'infirmités multiples très graves). De plus, les

\footnotetext{
${ }^{56}$ SHD, GR 7 NN 9 1037. Brouillon d'une note, Paris, 26 décembre 1928, Guizard et Sciard pour le secrétariat général.

${ }^{57}$ SHD, GR 7 NN 9 1037. Note, Paris, le 31 mai 1930, Sciard et Guizard, pour le secrétariat général.

${ }^{58}$ SHD, GR 7 NN 9 1037. Note, 22 octobre 1930, le secrétaire général du Ministère de la guerre pour l'Étatmajor de l'Armée.

${ }^{59}$ Le Mutilé de l'Algérie, 4 mai 1930, p. 4.

${ }^{60}$ SHD, GR 7 NN 9 1037. Note d'analyse de l'État-major de l'Armée, 17 février 1932, «Pensions des ayants droit des militaires ».
} 
mutilés indigènes de ces territoires coloniaux ne jouissent pas des augmentations attribuées aux mutilés français bénéficiaires de la loi du 31 mars 1919 au cours des années 1920. Par contre, les mutilés français de ces territoires coloniaux touchent une pension similaire à celle des Français de la métropole.

Tableau n². Tableau des pensions d'invalidité des militaires français et indigènes des différents territoires de l'Empire (montants fixés en 1919 et 1920).

Sources : JORF, 2 avril 1919, p. 8891 ; JORF, 13 septembre 1920, p. 13473.

\begin{tabular}{|c|c|c|c|c|c|c|c|c|c|c|c|c|}
\hline & \multicolumn{3}{|c|}{ 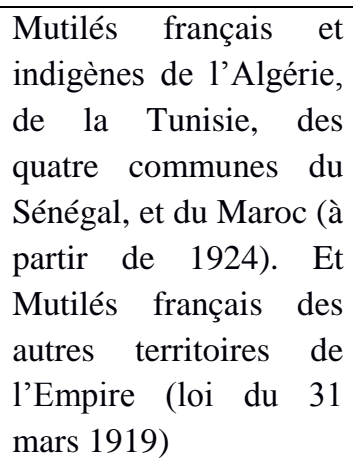 } & \multicolumn{3}{|c|}{$\begin{array}{l}\text { Militaires indigènes de } \\
\text { l'AOF et de l'AEF }\end{array}$} & \multicolumn{3}{|c|}{$\begin{array}{l}\text { Militaires indigènes de } \\
\text { l'Indochine, de la Côte } \\
\text { française des Somalis, } \\
\text { de la Nouvelle- } \\
\text { Calédonie, et des } \\
\text { Établissements français } \\
\text { de l'Océanie. }\end{array}$} & \multicolumn{3}{|c|}{$\begin{array}{l}\text { Militaires indigènes } \\
\text { de Madagascar }\end{array}$} \\
\hline $\begin{array}{l}\text { Pourcenta } \\
\text { ge } \\
\text { d'invalidit } \\
\text { é }\end{array}$ & $10 \%$ & $50 \%$ & $100 \%$ & $10 \%$ & $50 \%$ & $100 \%$ & $10 \%$ & 509 & $100 \%$ & $\begin{array}{l}10 \\
\%\end{array}$ & $\begin{array}{l}50 \\
\%\end{array}$ & $100 \%$ \\
\hline $\begin{array}{l}\text { Adjudant- } \\
\text { chef }\end{array}$ & 260 & 1300 & 2600 & 140 & 700 & 1400 & 113 & 565 & 1130 & 84 & 42 & 840 \\
\hline Soldat & 240 & 1200 & 2400 & 100 & 500 & 1000 & 72 & 360 & 720 & 60 & 30 & 600 \\
\hline
\end{tabular}

Les critères d'attribution des pensions sont très stricts et certains mutilés protestent contre le point de départ légal de la pension, l'évaluation de leurs pensions, le faible niveau des pensions, ou le non-renouvellement de leur pension. Ainsi, un tirailleur sénégalais mutilé nommé Doua-Bi-Daouan, résidant à Gouraoulfa (Côte d'Ivoire) amputé des deux mains et deux pieds, se voit refusé le renouvellement du bénéfice de sa pension d'invalidité en 1924 au motif qu'il a été atteint de gangrène des extrémités hors combat, lors d'une faction à Toulon. 
Informés de la situation, plusieurs fonctionnaires tentent d'alerter le gouverneur de l'AOF sur l'injustice de cette décision, susceptible de générer un « effet déplorable sur les indigènes ${ }^{61}$.

Les associations de mutilés réclament des augmentations régulières de leurs pensions au cours des années 1920, sans obtenir satisfaction. Les revendications des mutilés indigènes de ces colonies sont relayées par diverses associations de mutilés de la métropole (Union Fédérale, Association Républicaine des Anciens combattants), ainsi que ponctuellement par quelques parlementaires. En 1929, le député communiste de la Seine Georges Beaugrand s'insurge contre la faiblesse des pensions des mutilés indigènes (1000 francs pour un mutilé indigène $100 \%$ contre 7160 pour un mutilé français). Il se fait leur porte-parole en indiquant que les indigènes «attendent de toucher les mêmes rentes que les mutilés français » ${ }^{62}$.

Suite à la crise économique de 1929, l'État major de l'Armée se montre plus favorable au relèvement du taux des pensions d'invalidité et d'ancienneté des militaires coloniaux, du fait de l'intensité de la crise économique et sociale dans les colonies. Une commission interministérielle (Finances, Pensions, Guerre, Colonies) est constituée en 1932 pour étudier cette question et proposer de nouveaux tarifs. Elle approuve un principe essentiel : «la pension d'invalidité accordée au militaire indigène [a] un caractère essentiellement alimentaire - et que pour être équitable, elle [doit] être basée sur le coût moyen de l'existence de l'individu dans son milieu social ${ }^{63}$. Elle recommande d'augmenter l'ensemble des pensions, et d'accorder un complément pour les grands invalides indigènes. Le Ministère des Finances refuse d'accorder des augmentations aux pensionnés pour ancienneté, mais accepte d'augmenter les pensionnés pour invalidité. Un nouveau décret le 16 avril 1932 unifie et augmente le montant des pensions d'invalidité des militaires indigènes coloniaux (qui relèvent du décret de septembre 1920). Il délivre également des suppléments non négligeables pour les grands invalides nécessitant l'aide d'une tierce personne (à l'instar des suppléments prévus à l'article 12 de la loi de mars 1919) ${ }^{64}$. Il attribue enfin des droits égaux aux indigènes naturalisés français et aux Français, mais le nombre de bénéficiaires de cette dernière mesure est extrêmement restreint. Ces augmentations ne satisfont que partiellement les intéressés.

Tableau $n^{\circ}$ 3. Tableau des pensions d'invalidité des militaires indigènes non-officiers fixés par le décret d'avril 1932.

\begin{tabular}{|l|l|l|l|l|}
\hline Grades & $10 \%$ & $50 \%$ & $80 \%$ & $100 \%$ \\
\hline $\begin{array}{l}\text { Adjudant- } \\
\text { chef }\end{array}$ & 340 & 1700 & 2720 & 4000 \\
\hline tirailleur & 170 & 850 & 1360 & 2000 \\
\hline
\end{tabular}

Source : JORF, 22 avril 1932, p. 4345.

\section{3. Des ressources financières et un prestige suffisants pour fonder un foyer et connaître une ascension sociale?}

Ces pensions et les multiples bénéfices économiques connexes au statut de mutilé permettent aux mutilés français - et dans une moindre mesure aux indigènes - ayant un degré

\footnotetext{
${ }^{61}$ ANOM, 61 COL 192. Copie, Bingerville, E. Pieussergues, non datée (avant mars 1927), l'Administration du Cercle de Daloa au Gouverneur de l'AOF.

${ }^{62}$ Journal Officiel de la République française. Débats parlementaires, 28 novembre 1929, p. 3635.

${ }^{63}$ SHD, GR 7 NN 9 1037. Lettre, Paris, le 8 janvier 1937, le ministre des Colonies au ministre de la Défense.

${ }^{64}$ Journal Officiel de la République française, 22 avril 1932, p. 4345.
} 
d'invalidité important de vivre confortablement. Si la pension est d'un montant largement insuffisant pour les mutilés de la métropole (Prost, 1977, p. 56), elle s'avère d'un montant non négligeable pour les mutilés des colonies, pour lesquels elle constitue une précieuse rentrée d'argent liquide. La presse se fait l'écho de l'importance de ces ressources pour les indigènes : un observateur de Tlemcen (Algérie) indique : "Savez-vous que dans le bled le mutilé indigène est considéré comme un "mercanti", c'est-à-dire un privilégié de la fortune qui peut vivre sans travailler et goûter tout à son aise le "doux farniente" si cher aux Arabes ${ }^{65}$. Surtout, la pension leur offre la possibilité de subvenir aux besoins d'une famille, et donc de rester attractifs sur le marché matrimonial et de préserver leur masculinité dans un monde où l'homme a le devoir de subvenir aux besoins des siens. L'historien Echenberg mentionne d'ailleurs que le retour des soldats blessés dotés de pensions substantielles et pérennes a engendré «des redressements dans le prix d'achat des épouses » en AOF (Echenberg, 2009, p. 152). S'il nous est impossible de fournir des statistiques sur les taux de mariages, la lecture des revues associatives prouve que les mariages et les naissances sont fréquents parmi les mutilés. À titre d'exemple, l'aveugle de guerre tunisien Hassen Ben Salem Kriem, de Teboulba annonce en 1937 la naissance de sa fille Khedija à ses camarades de la métropole ${ }^{66}$. De plus, les mutilés européens et indigènes qui travaillent possèdent des ressources importantes grâce au cumul de la pension et du salaire. Ils peuvent ainsi constituer un petit capital, acheter une maison ou ouvrir un petit commerce (Bekraoui, 1987, p. 375). Toutefois, il ne faut pas surestimer cet apport financier de la métropole aux mutilés, qui n'intervient qu'épisodiquement (une fois par trimestre). De nombreux indigènes, ayant besoin de rentrées d'argent liquide plus régulières, contractent des prêts auprès d'usuriers, qui finissent par récupérer une partie non négligeable de la pension. En 1932, un groupe de mutilés de guerre indigènes de Mascara (Algérie) «proteste énergiquement contre l'exploitation honteuse dont la plupart d'entre eux sont victimes de certains usuriers qui leur font des avances sur leur pension à $150 \% »{ }^{67}$.

Toutefois, la principale interrogation est de savoir si les mutilés indigènes, grâce à ces ressources et au prestige conféré par le statut de mutilés, parviennent plus facilement à changer de statut juridique, à sortir de l'indigénat et/ou à devenir citoyen français dans les nouvelles colonies où cette distinction existe au cours de l'entre-deux-guerres. Dans les vieilles colonies, les quatre communes du Sénégal (Saint-Louis, Gorée, Dakar et Rufisque) et les établissements français de l'Inde, tous les habitants sont des citoyens français pendant cette période (Surun, 2012, p. 166). Dans les autres colonies françaises, les indigènes sont des "sujets français", non dotés de la citoyenneté française. Dans les pays sous protectorat, les indigènes sont des "protégés français", "sujets tunisiens" ou "marocains". De plus, dans certaines colonies (Algérie, AOF, AEF, Nouvelle-Calédonie, Madagascar, etc.), les indigènes sont soumis à des mesures discriminatoires liées au code de l'indigénat (infractions et punitions arbitraires, pouvant être transformées en travaux collectifs) (Fabre, 2010, p. 287).

Les indigènes mutilés de guerre, en tant qu'anciens combattants, figurent parmi les quelques catégories de personnes qui peuvent solliciter la naturalisation française. S'ils

\footnotetext{
${ }^{65}$ Le courrier de Tlemcen, 30 juin 1922, p. 1.

${ }^{66}$ Archives de la fondation des aveugles de guerre, Paris. Compte-rendu de la réunion du conseil d'administration de l'Union des aveugles de guerre du 24 avril 1937, p. 29.

${ }^{67}$ L'Echo d'Alger, 25 février 1932, p. 7.
} 
obtiennent la nationalité française, ils perdent leur statut d'indigène musulman et les contraintes afférentes. Le processus de naturalisation s'avère très aléatoire, les fonctionnaires décideurs appliquant de manière très restrictive et personnelle les mesures de naturalisation. En Algérie, suite à l'adoption de la loi Jonnart le 4 février 1919, les militaires indigènes ayant servi dans l'armée française ont la possibilité de demander individuellement la naturalisation française (Gros, 1997, p. 44). Les mêmes dispositions sont adoptées quelques années plus tard en Tunisie, en AOF, en AEF. Certains mutilés indigènes demandent effectivement à être naturalisés, comme le mutilé tunisien Mohamed El Ghardi, chaouch au contrôle civil de Sousse, qui sollicite sa naturalisation en $1923^{68}$. Il est soutenu dans sa démarche par l'Association générale des mutilés de la Grande Guerre en Tunisie. Nous ignorons si elle a abouti.

Toutefois, dans tous ces pays, l'administration distribue au compte-gouttes les certificats de nationalité française. Comme l'administration restreint considérablement le nombre des bénéficiaires, l'intéressé a plus de chances de l'obtenir s'il remplit d'autres critères (savoir lire et écrire le français, posséder une décoration militaire, être propriétaire, avoir exercé une fonction publique, avoir de bonnes moeurs, etc.). Au cours de l'entre-deuxguerres, quelques dizaines d'anciens combattants de l'AOF (dont de nombreux mutilés décorés) obtiennent ainsi leur naturalisation, mais un requérant sur deux est débouté (Coquery-Vidrovitch, 2001, p. 296).

La situation est légèrement différente à Madagascar, où les anciens militaires indigènes pensionnés ont la possibilité de demander à être exemptés de l'indigénat suite à l'adoption d'un arrêté d'exemption par le Gouverneur général en mars 1921 (Valensky, 1997, p. 79). Toutefois, cette sortie de l'indigénat ne les fait pas pour autant bénéficier du statut de citoyen français.

\section{CONCLUSION :}

Les mutilés de guerre sont en général considérés avec égards par les gouverneurs coloniaux et les responsables politiques métropolitains, qui adoptent dès 1916 une série de dispositions afin que ces mutilés ne se sentent pas "abandonnés" par la Patrie. C'est ce qui explique que les mutilés de guerre de l'Empire bénéficient d'une palette de droits presque similaires à ceux de la métropole (y compris des droits économiques) quelques années après ceux de la métropole, et non plusieurs dizaines d'années plus tard comme dans le cas des aveugles civils de l'Algérie (Brégain, 2016). Toutefois, les mutilés de l'Empire ne bénéficient pas des mêmes possibilités de rééducation professionnelle que ceux de la métropole ni de la gratuité des soins chirurgicaux et médicaux (exception faite de l'Algérie et de la Tunisie).Les sources restent insuffisantes pour mener une comparaison systématique de l'ensemble des prestations offertes dans tous les territoires, mais permettent de dresser le constat d'une extrême hétérogénéité des politiques d'assistance dans l'Empire colonial, avec de fortes inégalités territoriales et ethniques dans l'attribution des différentes prestations. Le statut privilégié accordé aux mutilés d'Afrique du Nord est perceptible à travers les circulations régulières de dirigeants associatifs et de responsables politiques (parlementaires mutilés,

\footnotetext{
${ }^{68}$ Bulletin trimestriel de l'Association générale des mutilés de la Grande Guerre en Tunisie, ${ }^{\circ} 19$, avril 1923, $\mathrm{p}$. 10.
} 
ministres des Pensions) entre la métropole et les pays d'Afrique du Nord, à travers les pensions élevées délivrées, et la présence importante de centres d'appareillage et de centres de rééducation.

La politique d'assistance aux mutilés de l'Empire constitue l'une des rares politiques sociales qui confèrent une protection pour les populations colonisées. Cette politique sociale, coûteuse pour la métropole, s'avère prioritaire en raison de l'impératif politique de reconnaissance envers ceux qui se sont sacrifiés pour la patrie, mais aussi et surtout pour conserver la sympathie des populations colonisées et le soutien politique des mutilés et des anciens combattants dans un contexte de nationalisme anticolonial croissant. Ces enjeux politiques expliquent l'égalité des pensions entre mutilés français et indigènes dans les pays d'Afrique du Nord et dans les 4 communes du Sénégal. Le cas du Maroc en est emblématique : le Général Lyautey est le plus réticent vis-à-vis du retour massif des mutilés indigènes en exigeant leur maintien en France ; il organise une politique massive de placement des mutilés de guerre; puis les autorités françaises acceptent de réintégrer les tirailleurs marocains dans l'armée régulière en 1924, leur permettant ainsi de bénéficier de l'égalité des droits avec les mutilés français sur le plan des pensions. À l'inverse, le choix des autorités françaises de conférer des droits nettement inférieurs aux mutilés de l'AOF (absence de politique de placement privilégié dans l'emploi, pensions moindres pour les indigènes), pourtant nombreux et reconnus pour leur bravoure, semble s'expliquer par leur perception de la situation politique de cette colonie, à savoir qu'il n'a pas de risques politiques à maintenir les hiérarchies coloniales et à discriminer ouvertement les mutilés indigènes de l'AOF.

En égalisant les droits des mutilés indigènes et français, cette politique sociale transgresse les hiérarchies coloniales, mais seulement de manière sélective sur le plan géographique (en Afrique du Nord et dans les quatre communes du Sénégal), et de manière partielle (uniquement sur le plan des pensions et de la rééducation professionnelle). De plus, une dizaine d'années après la fin de la guerre, l'empressement et l'intérêt témoignés à l'égard des mutilés indigènes se dissolvent, les réflexes discriminatoires rejaillissent dans les discours des hauts fonctionnaires du ministère de la Guerre, désormais prêts à rompre l'égalité de traitement des mutilés indigènes et français de l'Afrique du Nord sur le plan des pensions. Dans toutes les autres colonies, les mutilés indigènes sont fortement discriminés, ils ne disposent que d'une pension d'un montant largement inférieur à celle des mutilés français. Dans l'ensemble des territoires de l'Empire, les mutilés indigènes ont un accès restreint aux emplois administratifs, aux terres agricoles et aux prêts bancaires. Seule une très petite minorité d'entre eux réussit à sortir de l'indigénat et à se faire naturaliser français. Du fait du contexte colonial répressif, les mutilés indigènes n'émettent généralement que de manière indirecte - par la voix des mutilés français - leurs critiques de l'injustice des politiques coloniales. Si la condition de mutilé de guerre amène un grand nombre de mutilés français et indigènes à s'enorgueillir d'avoir servi la France, et à défendre les institutions françaises, d'autres, tel l'ancien tirailleur sénégalais mutilé Lamine Senghor dans la métropole (Murphy, 2013), combattent les injustices du système colonial au cours des années 1920.

\section{Références :}


Bekraoui, Mohamed (1987). Le Maroc et la Première Guerre mondiale, 1914-1920. Thèse de doctorat en Lettres, Université d'Aix-Marseille, sous la direction de Jean-Louis Miège.

Bette, Peggy (2006). Reclasser les victimes de la Première Guerre mondiale : le cas de la loi du 30 janvier 1923 sur les emplois réservés en France (1923-1939). Amnis. Revue d'études des sociétés et cultures contemporaines Europe-Amérique, nº6, 1- 10.

Brégain, Gildas (2016). Colonialism and disability : the situation of the Blind in colonised Algeria, ALTER, European Journal of Disability Research, vol. 10, 148-167.

Burbank, Jane et Cooper, Frédérick (2011).Empires. De la Chine ancienne à nos jours, Paris : Payot.

Collard, Clément (2018). Une institution exemplaire de la rééducation professionnelle des mutilés de guerre : l'Ecole Jean Janvier de Rennes dans l'entre-deux-guerres. En Envor, revue d'histoire contemporaine en Bretagne, $\mathrm{n}^{\circ} 12,1-15$.

Coquery-Vidrovitch, Catherine (2001). Nationalité et citoyenneté en Afrique occidentale français: Originaires et citoyens dans le Sénégal colonial. The Journal of African History, vol. $42, \mathrm{n}^{\circ} 2,285-305$.

Delaporte, Sophie (2001). Gueules cassées : Les Blessés de la face de la Grande Guerre, Noesis Agnes Vienot Eds.

Dimier, Véronique (2003). Décentraliser l'Empire ? Du compromis colonial à l'institutionnalisation d'un gouvernement local dans l'Union Française. Outre-Mers. Revue d'histoire, n³38/339, 83-107.

Echenberg, Myron J. (2009). Les tirailleurs sénégalais en Afrique-Occidentale française (1857-1960).Paris : Karthala.

Fabre, Martine (2010). L'indigénat : des petites polices discriminatoires et dérogatoires. In B. Durand, M. Fabre et M. Badji (dir.), Le juge et l'Outre-mer, Tome 5. Justicia illiterata : aequitate uti ? Les dents du dragon (pp. 273-310). Lille : Centre d'Histoire Judiciaire éditeur. Fichou, Jean-Christophe (2014). De l'irrationalité de la loi de 1923 sur l'emploi des mutilés de guerre dans le service des Phares et Balises. Annales de Bretagne et des pays de l'Ouest, $\mathrm{n}^{\circ} 121,147-165$.

Fogarty, Richard S. (2008). Race and War in France: Colonial Subjects in the French Army, 1914-1918. Baltimore: J. Hopkins University Press.

Fremeaux, Jacques (2006).Les colonies dans la Grande Guerre. Combats et épreuves des peuples d'outre-mer, Paris : 14-18 édition.

Ganiage, Jean (1994). Histoire contemporaine du Maghreb de 1830 à nos jours, Paris :

Fayard.

Gros, Dominique (1997). Sujets et citoyens en Algérie avant l'ordonnance du 7 mars 1944. Le Genre Humain, $\mathrm{n}^{\circ} 32,39-52$.

Hassett, Donal (2016). Pupilles de l'Empire. Debating the provision for child victims of the Great War in the French Empire. French Historical Studies, vol. 39, n² 2, 315-345.

Jobert, Bruno et Muller, Pierre (1987). L'État en action, politiques publiques et corporatismes. Paris : PUF.

Lagroye, Jacques ; François, Bastien ; Sawicki Frédéric (ed.) (2006). Sociologie politique, Presses de Sciences Po et Dalloz, 5e édition. 
Lekeal, Farid (2014). Pacifier par le droit social ? L'applicabilité de la législation ouvrière française en Algérie, 1895-1921. In S. Mechat (dir.). Coloniser, pacifier, administrer, XIXeXXIe siècles (pp. 245-263). Paris : CNRS éditions.

Murphy, David (2013). Defending the 'Negro Race': Lamine Senghor and Black

Internationalism in Interwar France. French Cultural Studies, vol. 24, n², 161-173.

Prost, Antoine (1977). Les anciens combattants et la société française, 1914-1939. Paris :

Presses de Sciences Po.

Romien, Pierre (2005). À l'origine de la réinsertion professionnelle des personnes handicapées : la prise en charge des invalides de guerre. Revue Française des Affaires Sociales, $\mathrm{n}^{\circ} 2,229-247$.

Surun Isabelle (dir.) et alii (2012). Les sociétés coloniales à l'âge des Empires, 18501960.Neuilly : Atlande.

Thomas, Martin (2007). The French Empire between the wars. Imperialism, politics and society. Manchester : Manchester University Press.

Valensky, Chantal (1997). Soldats malgaches et culture française (fin du XIXe-première moitié du XXe siècle). Revue française d'histoire d'outre-mer, tome 84, n³15, 63-84.

Wanaïm, Mbark (2009). Le régime administratif des unités marocaines entre la Première et la Seconde Guerre. Un éclairage historique sur les origines inégalitaires des pensions des anciens combattants marocains. Cahiers de la Méditerranée, nº78, 317-334.

Pour citer cet article : Gildas Brégain, « Réintégrer sans modifier les hiérarchies coloniales ? Inégalités ethniques et territoriales dans les politiques d'assistance aux mutilés de guerre de l'Empire colonial français (1916-1939) », ALTER European Journal of Disability Research, vol. 4, 2019. https://doi.org/10.1016/i.alter.2019.09.002 